\title{
Intimsphäre: rechtsethische Überlegungen zu einem Thema aus dem Kodex des Deutschen Presserates*
}

\author{
von Wolfgang Wunden
}

\section{Ein wichtiges Problem der Rechtsetbik}

In unserer „informationsreichen Gesellschaft" gewinnen solche Normen sittlichen Handelns eine überragende Bedeutung, welche Information oder - wie man heute oft sagt - Daten betreffen. Denn „eine Gesellschaft existiert nur dort, wo es freie Kommunikation zwischen den Menschen gibt... Man könnte sogar sagen, daß

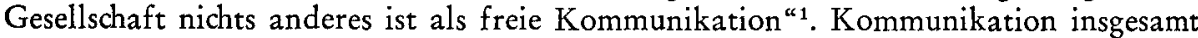
kann aber nur gelingen, wenn auch ihre Grenzen beachtet werden. Hierzu gehört die Wahrung dessen, was man heute "Intimsphäre“ nennt. Der Begriff „Intimsphäre“ greift zum Teil den traditionellen Terminus "Geheimnis" auf, fügt ihm aber neue Elemente hinzu.

D. de Soto, der den ersten großen Traktat über Geheimniswahrung verfaßte, war sich der Bedeutung dieses Gegenstands bewußt. Er schrieb: „Ein fremdes Geheimnis leichtfertig und ohne Grund $\mathrm{zu}$ offenbaren, ist generell eine Todsünde: zwar leichter als Mord und Ehebruch, aber schwerer als Diebstahl.“2

Seit de Soto hat allerdings diese Problematik neue Dimensionen erhalten; denn die Intimsphäre ist heute weitaus mehr bedroht als damals. Heute ist es beispielsweise mit Hilfe eines gut durchorganisierten Dienstleistungsapparates möglich, der Gesellschaft in kürzester Zeit jede beliebige Nachricht zukommen zu lassen. Massenmedien wie Rundfunk und Zeitungspresse, in Verbindung mit den modernen elektronischen Hilfsmitteln, bieten sich hierfür an. Die Bedrohung für den Einzelnen liegt darin, daß ein ganzer Dienstleistungsbereich damit beschäftigt ist, Daten zu sammeln und zu speichern, um sie bei Bedarf zu veröffentlichen. J.-L. Hébarre beschreibt das mit folgenden Worten: „Die immer ausgedehntere Tätigkeit der Informationsmittel bedroht mehr und mehr das Individuum in seinen wesentlichsten Rechten. Die großen Medien der sozialen Kommunikation haben in der Tat im Lauf der letzten Jahrzehnte einen solchen Aufschwung genommen, daß die Information fast allgegenwärtig ist, $\mathrm{da}$ sie im Verlauf ihrer normalen Informationssuche ständig ihren Bereich zu erweitern trachtet und einerseits in die weitentferntesten, andererseits in die geheimsten und intimsten Bereiche vorstößt. "3

Psychologie und Verhaltensforschung haben Methoden entwickelt, mit deren Hilfe man an gesicherte Informationen ïber den Intimbereich des Menschen kommen kann. Der Mensch ist in früher ungeahntem Ausmaß analysierbar.

Gleichzeitig wurden raffinierte technische Mittel erfunden, die es ermöglichen, in die Intimsphäre einzudringen. ${ }^{4}$ Die Informationen, die man auf diese Weise erhält, können zu guten und schlechten Zwecken benutzt werden. Die Computertechnik macht einerseits die gewonnenen Daten jederzeit verfügbar. Sie ermöglicht es, Daten, die etwa für das Gesundheits-, Sozial- oder Rechtswesen benötigt werden, abzurufen. Mit diesem großen Vorteil sind andererseits erhebliche Nachteile verbunden. So ist

Dr. Wolfgang Wunden ist persönlicher Referent des Intendanten des Süddeutschen Rundfunks und Mitarbeiter der Katholischen Akademie der Diözese Rottenburg. 
beispielsweise die Intimität des Einzelnen - von der hier zu reden ist - nicht mehr gesichert.

Trotz der offensichtlich großen Möglichkeiten, die Intimsphäre zu beeinträchtigen, ist das öffentliche Bewußtsein kaum mit diesem Gegenstand befaßt. So enthalten die drei Bände, in denen die Vorschläge der Interministeriellen Arbeitsgruppe beim Bundesministerium des Inneren für die Planung eines Informationsbankensystems für die BRD ${ }^{5}$ dargestellt und erörtert werden, zum Schutz der gespeicherten Daten nur Weniges. Wollte man aus der Zuschauerbeteiligung bei der Sendung „Datenschutz - Bedürfnis einer bedrohten Gesellschaft" (ZDF, Sonntag, den 9. September 1973, 22.05 Uhr) darauf schließen, wie bedroht sich die Gesellschaft tatsächlich fühlt, so muß man annehmen, daß dies kaum der Fall ist: nur 541.000 von 18.045.000 Geräten waren durchschnittlich eingeschaltet; die Zuschauerbeteiligung ging während der Sendung fast ständig zurück, und die gleichzeitig im ARD-Programm ausgestrahlte Sendung "Lebensläufe: Der Tippelbruder" hatte durchschnittlich $27 \%$ mehr Zuschauer. ${ }^{8}$ Gelegentliche Hinweise von Einzelpersonen, Orwells 1984 sei nicht weit entfernt, ändern an der geschilderten Situation nichts.

Sicherlich: in unserem freiheitlichen System scheint, auf den ersten Blick zumindest, die Intimsphäre im wesentlichen gesichert zu sein. Die Erinnerung an totalitäre Praktiken während des Dritten Reiches ist schon längst verblaßt, und 1984 scheint heute noch weit entfernt. Dem ließe sich entgegenhalten, daß bei uns das Gespür für Intimität verlorengegangen ist und deshalb auch große Verletzungen kein Gefühl der Bedrohung entstehen lassen. Dazu hat die Praxis unzähliger Starlets beigetragen, ihr Privatleben zu Publicity- $Z$ wecken zu verkaufen, ferner die Tatsache, daß gesellschaftliche Konflikte häufig in privatisierter Form dargestellt werden; auch die geschlechtliche Intimität ist weitgehend, etwa auf der Kinoleinwand, öffentlich geworden.

Dem Bewußtsein der Bevölkerung entspricht das geringe Maß an Interesse, das die Theologie dem Gegenstand entgegenbringt. Nur wenige Autoren befassen sich mit dem Thema, sei es unter dem Stichwort "Geheimnis" ${ }^{\text {"7 }}$, sei es unter dem Stichwort "Intimsphäre" .

Uber diesen mageren Befund kann auch die Tatsache nicht hinwegtrösten, daß das 2. Vatikanische Konzil den Schutz der Privatsphäre immerhin in seinem Katalog der Menschenrechte aufgenommen hat. ${ }^{{ }^{*}}$ Auch die gelegentliche Erwähnung durch Pius XII. (bei der Erörterung psychiatrischer Praxis) und Paul VI. ${ }^{10}$ (im $\mathrm{Zu}$ sammenhang der ethischen Probleme der Massenkommunikation) bleibt Episode, zumal da eine Ingerenz der „Amtskirche“ in die Intimsphäre von Betroffenen zuweilen beklagt wird. ${ }^{11}$

Die folgenden Gedanken zur Intimsphäre und zum Recht des Einzelnen auf ihre Achtung und ihren Schutz haben in dieser Lage unter anderem die Aufgabe, die Aufmerksamkeit aller Kreise auf dieses hohe Rechtsgut hinzulenken.

\section{Der Gegenstand des Rechts}

Eine Antwort auf die Frage zu geben, was Intimsphäre als Gegenstand eines Rechts eigentlich ist, stellt sich bei näherem Zusehen als gar nicht so leicht heraus. Eine Ubereinstimmung zwischen Autoren herbeizuführen, die den Terminus unterschiedlich begreifen, wird nicht einfach sein. H. Hubmann, anerkannte Autorität auf dem 
Gebiet des Persönlichkeitsrechts, wurde noch 1962 von G. Leinveber gerühmt, in seinem Werk "Das Persönlichkeitsrecht "12 den Begriff "Intimsphäre“ "rechtswissenschaftlich eindeutig abgegrenzt und klargestellt ${ }^{*} \mathrm{zu}$ haben. ${ }^{13}$ Er stellt aber in der 2. Auflage des gleichen Werkes ${ }^{14}$ kategorisch fest: "Der in der Literatur vielfach verwendete Begriff der Intimsphäre eignet sich nicht zur Umgrenzung eines Schutzkreises, weil er zu unklar ist". Dennoch scheint die Sache, um die es geht, wenn von Intimsphäre die Rede ist, so wichtig, daß es die Mühe lohnt, es trotzdem mit einer Definition zu versuchen.

Dabei kann man allgemeinere Umschreibungen - so legitim sie sind - außer acht lassen; wenn man etwa Intimsphäre als einen Bereich persönlicher Autonomie bezeichnet. ${ }^{15}$ Im Mittelpunkt unserer Betrachtung soll dagegen ein spezieller Aspekt des persönlichen Bereiches stehen: Intimsphäre nämlich, insofern sie Gegenstand von Geheimnisrechten und verwandten Rechten ist. Um diesen Aspekt eigens zu kennzeichnen, wird vorgeschlagen, den hier in Frage kommenden Bereich mit dem Neologismus „Intim“ (das Intim) zu bezeichnen.

Bei solchen Autoren, die sich mit diesem besonderen Aspekt der Intimsphäre befassen, erreicht das Intim sehr unterschiedliche Ausmaße: Für G. Arzt gehört das Geschäftsleben noch dazu, wenn auch nur „an der Peripherie" ${ }^{\text {10 }}$; H. Coing stellt identifizierend "den Bereich des Privatlebens oder die sogenannte Intimsphäre nebeneinander ${ }^{17}$, während $H$. Scholler das Intim auf die „Bereiche des Gewissens und der Liebe" beschränkt. ${ }^{18}$ Solche Unterschiedlichkeit in der Fassung des Begriffs kann nicht durch Dekret beseitigt werden. Immerhin ist bei allen Unterschieden festzuhalten, daß das Intim zum Privatbereich zu rechnen ist. Ferner läßt sich sagen, daß eine Tendenz besteht, Intimsphäre enger zu fassen als die gesamte Privatsphäre. Als Zeuge dafür läßt sich neben Scholler der Soziologe Habermas nennen. ${ }^{19}$

Der Theologe wird spätestens hier bemerken, daß ihm der Begriff "Intimsphäre“ eigentlich in Form der "intimitas" aus der Theologiegeschichte bekannt ist. Intimitas meint den Urgrund personalen Seins, die "Tiefe des Herzens“ als Mittelpunkt aller Strebungen des Menschen. ${ }^{20}$ So wenig die sprachliche Nähe beider Begriffe ein Kriterium für die Abgrenzung des Intim sein kann, so wenig sollte man verkennen, $\mathrm{da}$ sie eine enge Fassung nahelegt. Auch sachlich besteht hier ein Zusammenhang, der aber erst unten aufzuzeigen ist.

Bemerkenswert ist aus der Sicht der theologischen Ethik ein anderer Punkt, der die systematischen Prinzipien betrifft. Die traditionelle Ethik hat zwar Normen bezüglich verschiedener Geheimnisse gekannt: so schützte sie die Möglichkeit vertraulichen Austausches durch das sogenannte „secretum commissum", forderte, daß das Versprechen, etwas zu tun, auch gehalten wurde, wenn ein Geheimnis zu wahren versprochen worden war, kannte das Briefgeheimnis und ähnliche Normen. Ferner erschienen ihr Geheimnisse schutzwürdig, durch deren Bekanntwerden dem Betroffenen Schaden entstünde an Vermögen, Leben, Leib oder Seele, oder am guten Ruf oder in seiner Ehre (secretum naturale).

Davon aber, daß ein Geheimnis deswegen nicht bekanntgemacht werden darf, weil es zum Intim gehört, sprach die Moraltheologie nicht. Sie erfährt durch die moderne Rechtsentwicklung eine wesentliche Ergänzung und muß sehen, den Anschluß wiederzugewinnen. Allerdings sind hier bei Kuppens ${ }^{21}$ und vor allem bei Heylen ${ }^{22}$ Ansätze vorhanden (in seinem secret personnel), die beide eine Anregung aus dem klassischen Traktat über das Geheimnis von Kardinal de Lugo ${ }^{23}$ aufgreifen. 
Das so beschriebene Feld des Intim liegt ganz im Bereich des Geheimnisses und ist mit den bekannten genannten Kategorien (natürliches, persönliches Geheimnis) recht gut zu erfassen. Darïberhinaus gibt es nun weitere Vorgänge oder Situationen des Menschen, die zum Intim zählen, aber nicht mit dem "Geheimnis" zu erfassen sind. Einzelne oder mehrere Menschen befinden sich da in einer Situation, die man, in Anlehnung an Karl Jaspers, "Grenzsituation" nennen kann. Sie erfordert ein „intimes Fürsichsein", "wo alles Nahekommen im Grunde schon ein Eingreifen ist"24. Wir nennen sie „Situation mit Intimcharakter“.

Es gehört dazu, wenn sich ein Mensch in Unglück oder Verzweiflung befindet. Nicht wenige Autoren prangern die Neugier, Ehrfurchtslosigkeit und Erbarmungslosigkeit des Publikums in solchen Fällen an. ${ }^{25}$ Ebenso gehört dazu der ergriffene Mensch, besonders in der Begegnung mit Gott. ${ }^{2 \theta}$ Hierzu gehören auch Vereinigung, Gebären und Sterben - darüber wird unten noch zu sprechen sein.

Neben dem Intim in der Form des natürlichen oder persönlichen Geheimnisses und in der Form der Situation mit Intimcharakter muß auch generell und sozusagen quer zu diesen beiden der Bereich zum Intim gerechnet werden, den das Schamgefühl anzeigt. Selbst die divergierenden philosophischen Darstellungen des Schamgefühls und der Scham bei M. Scheler ${ }^{27}$ und O. F. Bollnow ${ }^{28}$ kommen darin überein, daß die Scham die Funktion des Verhüllens oder Verbergens hat: dies kommt bereits in der Grundbedeutung des Wortes "Scham" zum Ausdruck. Sie lautet: „sich bedecken "29. Von dieser sprachlichen Bedeutung her kann auf die Tatsache verwiesen werden, daß der Bereich, der durch das Schamgefühl als zu verhüllender angezeigt wird, von einigen Autoren "Intimbereich" genannt wird ${ }^{30}$; die Begriffe "Schambereich" und "Intimbereich" werden synonym verwendet. ${ }^{31}$ Dementsprechend wird der Wert, der durch Verhüllen aus Scham geschützt wird, als Intimwert bezeichnet. ${ }^{32}$

Das „Intim" umfaßt, wie aus diesen Beschreibungen deutlich geworden ist, neben Geheimnissen auch andere "Gegenstände" (Gegenstand hier im rechtsphilosophischen Sinn genommen). Letztere liegen oft vor allen offen, sind faktisch öffentlich, also nicht geheim oder verborgen.

\section{Kriterien christlicher Ethik}

Nachdem der Bereich des Intim umrissen ist, stellt sich die Frage, wie weit denn das Recht des Einzelnen in diesem Bereich geht. Das Recht nämlich, daß Geheimnisse gewahrt werden, die das Intim als Gegenstand haben; das Recht, in den beschriebenen Situationen mit Intimcharakter für sich zu sein und zu bleiben; das Recht, den Schambereich unberührt zu erhalten.

An diesem Punkt setzt die eigentlich moralische Fragestellung an: in welchem Aus$\mathrm{maß}$ und in welchen Grenzen ist das Intim Rechtsbereich? Oder: in welchem Ausmaß ist das Intim legitime Intimsphäre? ${ }^{33}$

Diese Frage setzt die Einsicht darin voraus, daß das Recht des Intim nicht unbeschränkt gilt, daß es vielmehr im Einzelfall in Konkurrenz zu anderen Rechten steht: Rechten, die unter Umständen - gerade im heutigen gesellschaftlichen Kontext - in der Regel den Vorrang beanspruchen: so zum Beispiel das Recht auf freie, ungehinderte Information.

Um bei Konflikten eine verantwortete Entscheidung fällen $z u$ können, ist erstens eine Einsicht in die funktionalen Zusammenhänge notwendig. Man muß - wenig- 
stens ungefähr - eine Einsicht darin besitzen, wozu Intimität generell gut ist; dann läßt sich ihr Wert mit anderen Werten vergleichen. $\mathrm{Zu}$ diesem Sachwissen kommen dann beim Christen zweitens theologische Motivationen hinzu. Sie verleihen dem durch die funktionale Analyse vermittelten Wert einen qualifizierten Anspruch darauf, verwirklicht zu werden. Die These, von der in folgendem ausgegangen wird, ohne daß sie hier näher begründet werden könnte ${ }^{34}$, lautet: Es gibt christliche Grundhaltungen, die - vermittelnd zwischen der Grundnorm personaler Selbstverwirklichung in Glaube, Hoffnung und Liebe und der ganz konkreten Norm für die einzelne sittliche Handlung - erwächst aus der christlichen Botschaft und dem Glaubensvollzug des Christen, in dem die Botschaft für sein Leben bestimmend wird. Damit halten wir fest, daß „im Offenbarungsereignis unaufgebbare anthropologische Gehalte einbeschlossen sind, die es als erstes und unmittelbarstes Kriterium aller weiteren positiven Normerhebung festzuhalten gilt. Es existiert, so verstanden, ein normatives Menschenbild des Evangeliums, in dessen Licht seine vielerlei Einzelaussagen aufzuspüren wie gleichzeitig zu bewerten sind "93. Diese Grundhaltungen ergeben insgesamt so etwas wie einen Mutterboden christlichen Handelns, dem die einzelnen Optionen verpflichtet sind. Sie sind dazu geeignet, im Konfliktfall Vorzugskriterien zu erbringen, die es dem Handelnden nahelegen, zum Beispiel auf eine Information bezüglich der Intimsphäre eines anderen zu verzichten, um diesem nicht sein Recht zu nehmen. Im folgenden werden - im Anschluß an die Schilderung funktionaler Zusammenhänge - einige dieser theologischen Motivationen genannt.

Ein erstes Beispiel könnte zeigen, wie eine legitime Intimsphäre zum Kernbestand menschlicher Freiheit gehört. Zwar ist der Mensch frei, „und wär er auch in Ketten geboren ", aber es muß über diese geistige Freiheit hinaus auch einen Freiheitsraum geben, damit von wirklicher menschlicher Freiheit gesprochen werden kann. Eine Analogie im Bereich von Geheimnissen mag verdeutlichen, um was es hier geht.

Geheimnisse, die ein Kind bewußt vor den Eltern hat, sind die unentbehrlichen Stïtzen zur Loslösung von den Eltern. „In dem Maße, wie es die Freiheit besitzt, ihnen seine Geheimnisse zu verschweigen, kommt ihm seine Eigenartigkeit zum Bewußtsein, erkennt es, daß es sich von ihnen unterscheidet, daß es Person ist. " ${ }^{\text {"e }}$ Wenn der Glaube an das elterliche Allwissen aufhört, entsteht im Kind ein ganz neues Gefühl von Macht.

An diesem Beispiel wird deutlich, wie wichtig für die Selbstfindung und - konkreter - für das Bewußtsein, jemand zu sein, „ich“ zu sein, es ist, Geheimnisse zu haben. Wo dies auf die Dauer verhindert wird, werden schwere Schädigungen nicht auf sich warten lassen, die auf einen Mangel an Selbstand hinauslaufen mit allen Konsequenzen, die dieser hat. In späterer Zeit wird es ähnlich sein: ein Erwachsener, der keine persönlichen Geheimnisse haben darf, ist des Raumes beraubt, in dem sich verantwortliche Stellungnahme entfalten kann.

Damit wird ihm nämlich ein kontrollfreier Raum entzogen. Das belastet ihn besonders dann, wenn von ihm die Umwelt ein bestimmtes Verhalten erwartet, wenn er also, falls sein Verhalten den Erwartungen nicht entspricht, mit Sanktionen zu rechnen hat. Er wird aus Furcht vor entstehenden Konflikten sein Verhalten yeitestgehend den Erwartungen anpassen.

Weiterhin ist die Intimsphäre nötig als ein Raum, in dem der Mensch ungehindert lernen $\mathbf{k a n n}^{37}$, ohne gleich mit allen Konsequenzen festgelegt $\mathrm{zu}$ sein. 
Alles in allem zielt die Wahrung der Intimität auf einen selbstbewußten, im Urteil reifen Menschen hin, dessen Uberzeugungen in einem Prozeß der Identitätsfindung entstanden sind.

Zur Intimität im Dienst der Selbstwerdung und Selbstfindung sollte der Christ aus der Einsicht in die Selbsterschließung Gottes in der Botschaft Jesu Christi und alles damit Gesagte - ein besonderes Verhältnis haben. Auf freie Zustimmung ist Gottes Botschaft angelegt, Christen glauben ihn als den personalen Gott und den Grund kreatürlicher Freiheit; Jesu Autorität appelliert an menschliche Freiheit, seine Zeichen zwingen den Menschen nicht, sondern setzen ihn frei von Z,wängen und sind Medium, in dem er sich den Menschen offenbart. Jesus ruft die Menschen zur Umkehr, weil er glaubt, daß mit der Gnade Gottes der Mensch "umkehren“ kann; der Mensch ist mit wachsender personaler Einsicht mehr und mehr verantwortlich für sein Tun. Um solche Freiheit zu gewinnen und zu bewahren, braucht der Mensch Intimität. Deswegen geht Jesus selber immer wieder allein „für sich“, „an einen einsamen Ort“, ehe er in seiner Hingabe den höchsten Akt seiner Freiheit setzt.

Zudem ist das Geheimnis persönlichsten Seins - hier sind wir auf einer ganz bestimmten engen Stufe der Intimsphäre - Medium personaler Nähe. Doch wollen wir das am Beispiel des Intimbereichs der Scham zeigen.

Oben wurde der Schambereich als ein Teil des Intim beschrieben. Welche Aufgabe hat nun das Schamgefühl? Welchen Sinn der Wille, bestimmte Bereiche zu verhüllen?

Nun, wir meinen - ohne dies hier weitläufig begründen zu können - daß das Schamgefühl eine wertvolle Hilfe im Aufbau und im Bestand differenzierter menschlicher Beziehungen ist. Es ermöglicht erlebte menschliche Nähe. Immer ist der Scham eine Furcht mitgegeben. Wer sich schämt, fürchtet sich, sich zu enthüllen, sich dem anderen bloß zu zeigen. Diese Furcht verhindert, daß einer unterschiedslos mit allen in die gleiche ,intime " Beziehung tritt. Sie hilft, den Augenblick abzuwarten, in dem eine Beziehung dazu reif ist, eine für diese Menschen einzigartige zu werden. Insofern hilft die Furcht in der Scham, personale Beziehungen aufzubauen und zu gestalten.$^{38}$ Dies gilt gleicherweise für den geistigen wie für den körperlichen, vorwiegend erotisch geprägten Intimbereich.

In diesem Sinne wären auch die zutreffenden Bemerkungen zu präzisieren, die A. Elsässer zum personalen Charakter der Intimsphäre macht. Er sagt: „Nicht das Geschlechtliche als solches (wird) von der Scham geschützt..., sondern das Menschliche an der Geschlechtlichkeit. Oder anders ausgedrückt: das Geschlechtliche wird von der Scham geschützt, weil es menschlich, d.h. personal durchformt, von der seelisch-geistigen Liebe getragen und selbst leibhaft sinnenfälliger Ausdruck dieser Liebe ist, weil er wesentlich ausgerichtet ist auf das $D u, d$. h. auf den anderen, nicht als reines Geschlechtswesen, sondern als Person und Partner. " ${ }^{\text {"39 }}$ Scham ermöglicht, zu einem anderen in eine einzigartige Beziehung $z \mathfrak{u}$ treten, ihn erst $z \mathfrak{u}$ einem $D \mathfrak{u}$ in einem ganz prägnanten Sinn zu machen; neben dem es - zumindest zeitweilig niemand anderen gibt.

Christliche Botschaft gibt auch hier starke Impulse. Sie kennt keine unterschiedslose Gleichheit (es sei denn die paulinische, daß alle sündigen und der Gerechtigkeit Gottes bedürfen). Die Bibel weiß von großen, innigen Freundschaften (David und Jonathan, Jesus und Johannes), der Erwählungsgedanke ist ein durchgängiges zentrales Thema der Offenbarung. Die bräutliche Liebe ist ihr ein Bild solcher Innigkeit, 
daß es die eschatologische Nähe Gottes bezeichnen kann. Das trinitarische Dogma schließlich denkt die Personalität Gottes als einzigartiges Im-Austausch-Sein. Der Rechtsgehalt des Intimbereichs der Scham liegt zweifellos im Maß der Nähe zwischen Menschen begründet, die das Schamgefühl ermöglicht. In diesem Maß ist es auch von christlicher Botschaft getragen.

Wieder ein anderer Sachverhalt ist auf je eigene Weise in den Situationen mit Intimcharakter anzutreffen. Es geht um den Bereich, in dem Takt, Höflichkeit, ja Konventionen gelten. Konventionen sind ja nicht nur erstarrte Floskeln; sie haben auch, recht verstanden und gebraucht, eine Stützfunktion. Sie ermöglichen es dem Einzelnen, sich ohne unangenehme Belästigung bewegen und leben zu können. So gibt es den Händedruck als nichts über die Art der Beziehung sagendes Medium der Begruißung, den Gruß mit Worten als Modus des Aneinander-Vorbeigehens oder der Gesprächsanknüpfung. Sie sind ein Mittel, mit dem man Distanz und Nähe regeln kann. So sehr sie sich auf der Ebene des Unverbindlichen halten, so notwendig sind sie, damit nicht der Mensch ständig im Nähe-Darbieten überfordert ist oder aber die deswegen erforderliche Distanz als Abweisung verstanden wird.

Besonders braucht der Mensch Distanz, - eine Distanz, die Teilnahme keineswegs ausschließt, wenn sie nur "diskret ${ }^{\alpha}$ ist -, wenn ihn etwas tief bewegt, ja überwältigt. Manchmal hat er die Möglichkeit, in solchen Situationen, sich zu verbergen - hinter Schweigen, belanglosen Worten, gestalteten Gebärden, vielleicht in einem Raum usf. - , manchmal hat er diese Möglichkeit nicht. Gerade um diesen letzteren Fall geht es in den Situationen mit Intimcharakter. Der Mensch kann sich nicht (mehr) beherrschen, ist seiner (und dies drückt sich körperlich aus) nicht mächtig."0 Hier hat er nicht die Möglichkeit, sich hinter der Konvention zu verbergen oder auf andere Weise für sich zu sein. Hier muß der andere und das Publikum von sich aus das Intim respektieren: nicht neugierigteilnahmslos gaffen, im Bild festhalten, auf Tonband aufnehmen.

Auch in diesem Bereich läßt sich durchaus eine christliche Wertschätzung der Intimität aufzeigen. Das Anschauen und Nichtbedecken des trunkenen Noe wird in der Bibel als Frevel gewertet; und wer meint, hier habe man es lediglich mit kulturellen Standards zu tun, die heute nicht mehr gelten, muß sich Jesu Vorwurf anhören: "Ich war nackt, und ihr habt mich nicht bekleidet"; und das Reich duirfen die in Besitz nehmen, die ihn nicht nackt gelassen, sondern bekleidet haben (Mt 25).

\section{Bemerkungen zur journalistischen Berufsethik}

Hiermit ist das Rechtsphänomen "Intimsphäre" in seinen wesentlichen Zügen umrissen, und Vorzugskriterien sind, wenigstens schwerpunktmäßig, angegeben. Welcher Wert - die Intimsphäre oder das mit ihr im Wettbewerb liegende Gut - im konkreten Fall den Vorzug erhält, ist damit zwar noch nicht entschieden - kommt es doch u. a. auf die Bedeutung des konkurrierenden Wertes an, der höher oder niedriger stehen mag im Vergleich mit dem jeweiligen Rang des Intimwertes. Im voraus zu bestimmten Einzelfällen kann man aber Zuordnungen von Intimwert und anderen Werten feststellen. Das sei - wegen der großen Bedeutung des Problems in unserer Gesellschaft - am Beispiel journalistischer Deontologie aufgezeigt. Einem guten Journalisten, dessen Beruf es ist, Informationen zu sammeln und zu verbreiten, wird es trotzdem gelingen, an der Grenze „Intimsphäre" Halt zu machen. Dazu braucht er neben Taktgefühl und Einfühlungsvermögen die Uberzeugung, daß beide Rechte letztlich auf dasselbe Ziel hingeordnet sind. Das Recht, 
informiert zu werden, gibt dem Einzelnen die Möglichkeit, ein freier und verantwortlicher Mensch zu werden und als solcher am Leben der Gesellschaft teilzunehmen. (Der Journalist ist dabei der Sachwalter dieses Rechts). Demselben Ziel dient das Recht des Einzelnen auf seine Intimsphäre. An diesen Sachverhalt hatte wohl auch Papst Paul gedacht, als er bemerkte, die Grenzen legitimer Information dürften nicht willkürlich gezogen werden, sondern sie müßten unmittelbar mit der pehrenhaften Sendung " der Massenkommunikationsmittel zusammenhängen und von da her als sinnvoll erwiesen werden. ${ }^{41}$

Unter Verstößen in diesem Punkt haben besonders die sogenannten „Personen der Zeitgeschichte ${ }^{\alpha}$ zu leiden. ${ }^{42}$ Es dürfte klar geworden sein, daß berühmte Leute ebenso das Recht auf die Intimsphäre besitzen wie jeder andere. Wer meint, so ein Mensch bezahle seine Berühmtheit mit dem Verzicht auf die Intimsphäre, macht eine falsche Rechnung auf. Und ferner: der Einwand, eine Person des Zeitgeschehens finde mehr legitimes Interesse auch bezüglich seiner Intimsphäre als Lieschen Müller, trifft zwar etwas Richtiges; aber ebenso gilt, daß öffentliches Interesse etwas ganz anderes ist als jedermanns Neugier.

Doch selbst dann, wenn man der Information den Vorzug vor der Intimsphäre gibt, kann man den Intimwert immer noch achten. Muß man einem anderen „zu nahe treten", so kommt es sehr darauf an, wie man es macht.

Es wird vieler Mühe bedürfen, um heute die Intimsphäre aller Menschen angemessen zu schützen. Der "Regierungsentwurf eines Gesetzes zum Schutz vor Mißbrauch personenbezogener Daten bei der Datenverarbeitung" (Drucksache 7/1021 vom 21.9. 1973) ist deswegen - vor aller Detailkritik daran - als ein weiterer wichtiger Schritt zu begrüßen. Theologische Ethik wird es dabei als ihre besondere Aufgabe verstehen, immer wieder herauszustellen, warum die Intimsphäre zu schützen ist und welchen inneren Sinn dieser Freiheitsraum hat. Was oben schon ausgeführt wurde, faßt auf seine Weise ein Gedanke Mihajlovs aus dem eingangs zitierten Brief zusammen. Sein Briefpartner Quadrelli meint, der Westen sei an seiner Freiheit zugrundegegangen. Er erhält folgende Antwort: „Man könnte sagen, daß das, was Du als Zerfall des freien Westens bezeichnest, die Existenz einer wachsenden Zahl von innerlich unfreien Menschen voraussetzt. Kommunikation ist unter Menschen nur möglich als Ausdruck der Tiefen des ,Ich' eines jeden, das heißt der Fundamente einer Person, der Seele und jener seelischen Bereiche, die nicht zur sichtbaren physischen Realität gehören. Wenn sich ein Mensch von seinem fundamentalen ,Ich loslöst, wird es ihm unmöglich, mit einem anderen Menschen Verbindung aufzunehmen. Freiheit aber ist genau die ungebrochene Verbindung mit diesem ,Ich'."

\section{Abkürzungen:}

ECatt = Enciclopedia Cattolica, Rom $1949 \mathrm{ff}$.

EThL $=$ Ephemerides Theologicae Lovanienses, Brügge $1924 \mathrm{ff}$.

LThK = Lexikon für Theologie und Kirche, Freiburg i. Br. ${ }^{2} 1957 \mathrm{ff}$.

AAS $=$ Acta Apostolicae Sedis, Rom 1949.

LPäd(F) = Lexikon der Pädagogik, I-IV, Freiburg i. Br. 1952-1955.

\section{Anmerkungen:}

" In seinen „Publizistischen Grundsätzen " vom 12. 12. 1973 kodifiziert der Deutsche Presserat: „7. Die Presse achtet das Privatleben und die Intimsphäre des Menschen. Berührt jedoch das private Verhalten eines Menschen öffentliche Interessen, so kann es auch in der Presse erörtert werden. Dabei ist zu prüfen, ob durch eine Veröffentlichung Persönlichkeitsrechte Unbeteiligter verletzt werden." 
1. M. Mihajlov: Brief an Quadrelli, in: „Die Welt“, 29.9.1973, Beilage Die geistige Welt, I.

2. D. de Soto: De ratione tegendi, et detegendi secretum, Brixen 1582, S. 26.

3. J.-L. Hébarre: Protection de la vie privée et déontologie des journalistes, Zürich 1970, S. 13 ,

4. Vgl. das erste Kapitel von R. Kamlah: Right of Privacy. Das allgemeine Persönlichkeitsrecht in amerikanischer Sicht unter Berücksichtigung neuer technologischer Entwicklungen, Köln 1969; ferner H. Greulich: Spion in der Streichholzschachtel, Gütersloh 1969.

5. Das Informationsbankensystem. Vorschläge für die Planung und den Aufbau eines allgemeinen arbeitsteiligen Informationsbankensystems für die Bundesrepublik Deutschland, Köln 1971/72.

6. Infratam-Wochenbericht 36:1973.

7. G. Ermecke: Uber Pressefreiheit und Presseverantwortung. Pressefreiheit und Geheimniswahrung, Paderborn 1965. (Zur Intimsphäre äußert sich E. nur kurz, S. 43 f.); G. B. Guzzetti u. a.: Segreto, in: ECatt XI 252-264. A. Lanza - P. Palazzini: Theologia moralis, Bd. $2 / 2$, Turin-Rom 1965, S. 911-933. Zu grundlegenden Überlegungen führen ein älterer Artikel von M. Kuppens: Le respect du secret naturel, in: „Rev. ecclés. de Liège $33: 1946,181-187$, und folgende Arbeiten von V. Heylen: De Jure et Justitia, Mecheln 1950; Een geheim hebben of een geheim zijn, in: "Hospitalia“ $7: 1962,27-29$; Spécification morale des obligations interpersonnelles objectives dans la législation sociale actuelle, in: EThL 40:1964, 362-390.

8. B. A. M. Peters: La valeur morale de l'intimité personnelle. Un probleme actual posé à propos de l'influencement pharmacologique délibérément exercé sur la personnalité, in: "Studia Moralia", Bd. 2, Rom u. a. 1964, S. 191-254; R. Egenter: Intimsphäre, in: LThK $^{2}$ V 736 f.; A. Elsässer: Die persönliche Intimsphäre, Augsburg 1969; R. Bleistein: Sexualerziehung zwischen Tabu und Ideologie, Würzburg 1971, darin vor allem der Abschnitt „Intimsphäre und Freiheit", S. 111-117.

9. Pastoralkonstitution „Gaudium et Spes“, Nr. 26,2.

10. Pius XII.: Ansprache an die Teilnehmer des 13. Kongresses für angewandte Psychologie, in: AAS 50:1958, 268-282; Paul VI.: Ansprache an die Teilnehmer des Seminars der Vereinten Nationen über die Freiheit der Information, in: AAS 56:1964, 387-379; Brief des Kardinalstaatssekretärs A. G. Cicogniani vom 8.11.1968 an die 4. Vollversammlung der katholischen Presseunion Italiens (U.C.S. I.) in Tarent, mit Anregungen und Wünschen Papst Pauls VI., in: "Communicatio socialis" 2:1969, 152-157; die Hinweise auf Paul VI. bei G. Deussen: Ethik der Massenkommunikation bei Papst Paul VI., München u. a. 1973, S. 126 f.

11. Etwa bei Eheprozessen, oder im Zusammenhang mit den Normen für die Laisierung von Priestern (vgl. A. Dordett: Ubberlegungen zur Rückführung in den Laienstand, in: "Diakonia / Der Seelsorger" 2:1971, 278-280).

12. H. Hubmann: Das Persönlichkeitsrecht, Köln 1953.

13. G. Leinveber: Zur Frage der Verletzung der „Intimsphäre“ (Persönlichkeitsrechtsverletzung und ihre Folgen), in: "Jur. Rundschau" 1962, 164-166 (166); ebenso ders.: Zum Begriff der "Intimsphäre“, in: „Gewerbl. Rechtsschutz und Urheberrecht" 63:1961, 274$276(274)$.

14. H. Hubmann: Das Persönlichkeitsrecht, Köln 1967.

15. 1965 wurde in einem Staat der USA unter Berufung auf das dem Recht auf die Intimsphäre entsprechende "right of privacy" das Recht erstritten, in der Ehe antikonzeptionelle Mittel zu verwenden (Griswold v. Connecticut; vgl. A. Simmel, Privacy, in: International Encyclopedia of the Social Sciences, Bd. 12, New York 1968, 486); ähnlich vertritt K. Rahner die Auffassung, daß der Bereich der Zeugung zur legitimen Intimsphäre gehöre und von staatlichen Eingriffen frei bleiben müsse (Zum Problem der genetischen Manipulation, in: Schriften zur Theologie, Bd. 8, Einsiedeln-ZürichKöln 1967, S. 313).

16. G. Arzt: Der strafrechtliche Schutz der Intimsphäre, Tübingen 1970, S. 131.

17. H. Coing: Die heimliche Tonbandaufnahme als Rechtsproblem, in: Tonbandaufnahmen, Zulässigkeit und Grenzen ihrer Verwendung im Rechtsstaat. Bericht über eine Arbeitstagung (Weinheimer Tagung, einberufen vom Institut zur Förderung öffentlicher Angelegenheiten), Mannheim 1957, S. 36. 
18. H. Scholler: Person und Offentlichkeit. Zum Spannungsverhältnis von Pressefreiheit und Persönlichkeitsschutz, München 1967.

19. Für Habermas hebt sich der kleinfamiliale Intimbereich vom privaten Bereich des Warenverkehrs und der gesellschaftlichen Arbeit ab: Strukturwandel der Offentlichkeit, Neuwied ${ }^{4} 1962$.

20. H. Schlier versucht eine Beschreibung: Das Menschenherz nach dem Apostel Paulus, in: ders.: Das Ende der Zeit, Freiburg u. a. 1971, S. 184-200, bes. $199 \mathrm{f}$.

21. S. oben Anm. 7.

22. S. oben Anm. 7.

23. J. de Lugo: De Justitia et Jure, Bd. 1, Venedig 21751, bes. S. 239.

24. N. Hartmann: Ethik, Berlin ${ }^{4} 1962$, S. 478.

25. A. Böhm: Das Zeitalter der Indiskretion, in: „Wort und Wahrheit" 9:1954, 181-193; J. Streuli: Presse, Information, Sensation, in: "Schweizer Monatshef te" $44: 1954 / 65,211-$ 218; F. Sieburg: Haben wir noch ein privates Leben?, in: „Universitas" $8: 1953,663 \mathrm{f}$; E. Schmidt: Der Einbruch in die Intimsphäre in juristischer Sicht, in: "Universitas" 13:1958, 813-825 (abgedruckt in: Freiheit der Persönlichkeit, Stuttgart 1958 (Kröners TB-Ausgabe Bd. 290) S. 131-150); ders.: Offentlichkeit oder Publicity? in: Aktuelle Probleme aus dem Gesellschaftsrecht und anderen Rechtsgebieten (Festschrift für Walter Schmidt), Berlin 1959, S. 338-353; E. Dovifat: Pressefreiheit und Schutz der Ehre und der Intimsphäre, in: Universitätstage 1959 der Freien Universität Berlin, S. 36-46; W. Schöllgen: Das Neue und die Sensation: das menschliche Informationsbedürfnis auf dem Hintergrund der philosophischen Anthropologie, in: ders.: Konkrete Ethik, Düsseldorf 1961, S. 286-299.

26. H. B. Meyer: Zeitprobleme christlich gesehen, Bd. 1, Stuttgart 1969, S. 27.

27. M. Scheler: Uber Scham und Schamgefühl, in: $G W X=$ Schriften aus dem Nachlaß Bd. 1: Zur Ethik und Erkenntnislehre, Bern 21957, S. 65-154; ders.: Die Ehrfurcht, in: Vom Umsturz der Werte, GW III, Bern ${ }^{4} 1955$, S. 26-31.

28. O. F. Bollnow: Die Ehrfurcht, Frankfurt ${ }^{2} 1958$.

29. F. Kluge - A. Götze: Etymologisches Wörterbuch der deutschen Sprache, Berlin ${ }^{15} 1951$, 650, Art. Scham.

30. Herders Fremdwörterbuch, Freiburg 1969, 207: „Intimsphäre...: der durch natürliches Schamgefühl gg. Dritte geschützte bzw. zu schützende Bereich..."

31. H.-J. Pfistner: Uber das Schamgefühl in psychoterapeutischer und heilpädagogischer Praxis, in: "Wege zum Menschen“ 14:1962, 49-58: "Schambereich (Intimsphäre)" (57).

32. W. J. Revers: Scham, Schamgefühl, in: LPäd (F) III 1205; L. M. Weber: Scham, Schamhaftigkeit, in: LThK ${ }^{2}$ IX 365.

33. Mit dieser Formulierung wird der Unterschied zwischen dem deskriptiven Begriff "Intimsphäre" und dem normativen Begriff "Intimsphäre" angezielt. Manche Verwirrung in der Diskussion ließe sich vermeiden, wenn man sich diesen Unterschied immer vor Augen hielte.

34. Vgl. dazu im einzelnen K. Demmer: Moralische Norm und theologische Anthropologie, in: "Gregorianum ${ }^{c} 54: 1973,263-306$.

35. K. Demmer: a.a.O. 290.

36. P. Tournier: Jeder hütet sein Geheimnis, Zürich und Stuttgart, 1965, S. 10.

37. Vgl. K. W. Deutsch: Politische Kybernetik. Modelle und Perspektiven, Freiburg ${ }^{2} 1970$, S. 196.

38. Eine wertvolle Anregung zu dieser Uberlegung verdanken wir Thomas von Aquin: Summa theologia I-II q. 44 a. 4: "Utrum timor impediat operationem". Er antwortet darauf: "Eine Furcht, die maßvoll ist, trägt dazu bei, daß man richtig handelt; denn sie erzeugt "Aufmerksamkeit und Besorgtheit und erreicht, daß der Mensch sich mehr Gedanken macht und dann erst überlegt handelt."

39. A. Elsässer: Die persönliche Intimsphäre, S. 99.

40. Damit hat Thomas von Aquin (in Anlehnung an Aristoteles) den Intimcharakter der menschlichen Sexualorgane begründet: der starke Sexualtrieb, "überwältigt" die (ausgeglichen-souveräne) Vernunft, die an sich und normalerweise im Menschen dominiert. 
Der Mensch ,schämt sich“ wegen dieser Entthronung seiner Vernunft; vgl. J. Fuchs: Die Sexualethik des heiligen Thomas von Aquin, Köln 1949, bes. S. 42-50.

41. In: AAS 56:1964, 387.

42. Darauf hat zu Recht Kardinal Suenens in einer Intervention am 24.11.1962 beim 2. Vatikanischen Konzil hingewiesen, s. Acta Concilii Vaticani Secundi, Periodus I, congregatio generalis 26,463 .

\section{S UM M A R Y}

The right to keep personal secrets and related matters belong to the basic right of human intimity. It has always been central to human communication, but much more today in a cultural environment of technical possibilities and overflow of information. This right needs special treatment by the ethics of law. To be able to judge the extent of this human right, criteria have to be spelled out - for this the functional context should be described. Theological ethics over and above can describe criteria derived from Christian message and faith. Single cases have to be decided in this perspective, and journalists should benefit from this for their work.

\section{RESUMÉ}

Le droit au secret personnel et, avec lui, les droits analogues appartiennent aux droits les plus essentiels de la sphère intime. Depuis toujours ils sont au centre de la communication humaine et dans le contexte culturel actuel qui se caractérise par l'abondance d'informations et par la perfection des possibilités techniques ils exigent une attention renforcée du côté de l'éthique juridique.

Il faut nommer des critères de préférence pour définir jusqu'où vont ces droits. A cela s'ajoutent des rapports fonctionnels qui seront définis. L'éthique théologique peut à partir de cela présenter des critères qui sont compris dans le message chrétien et l'accomplissement de la foi qui lui est uni.

Les cas particuliers dans les domaines spécialisés essentiels doivent être résolus dans cette perspective. Les journalistes y gagnent de proportions éthiques pour leur travail.

\section{RES UMEN}

Entre los derechos esenciales de la esfera íntima se encuentran el derecho al secreto personal y los derechos con éste emparentados. Siempre fueron aspecto central de la comunicación humana, pero en el contexto cultural de hoy, caracterizado por la abundancia de información y la perfección de las posibilidades técnicas, exigen una mayor atención por parte de la ética jurídica. Para precisar en qué medida son suficientes tales derechos deben fijarse criterios de prioridad. Para ello se describen los contextos funcionales. La ética teológica puede además aportar criterios incluídos en el mensaje cristiano y en la consumación de la fe que le está unida. Bajo esta perspectiva deben ser decididos los casos particulares en materias relevantes. Los periodistas logran así normas éticas para su trabajo. 